\title{
Surface term for the capillary condensation transitions in a slit geometry
}

\author{
Ignacio Urrutid * and Leszek Szybist* \\ Laboratorio TANDAR, Departamento de Física, Comisión Nacional de Energía Atómica, \\ Av. del Libertador 8250, RA-1429 Buenos Aires, Argentina and \\ Departamento de Física, Facultad de Ciencias Exactas y Naturales, \\ Universidad de Buenos Aires, Ciudad Universitaria, RA-1428 Buenos Aires, Argentina
}

(Dated: August 12, 2018)

\begin{abstract}
It is shown that a bare simple fluid model (SFM) proposed some years ago for studying adsorption between two semi-infinite solid walls can be improved by modifying the surface term in the grand potential for the film phase. Such a correction substantially improves the agreement between the predictions for phase transitions provided by that SFM and results obtained from calculations carried out for ${ }^{4} \mathrm{He}$ with the density-functional method at zero temperature. The corrective term depends on the strength of the adsorption potential and observables of bulk helium.

PACS numbers: 61.20.-p, 64.70.-p, 67.70.+n
\end{abstract}

A simple fluid model (SFM) has been proposed by Gatica et al. [1] for exploring the behavior of adsorption between two parallel walls separated by a distance $L$. In that paper, transitions between empty (E), film $(\mathrm{F})$, and capillary condensation $(\mathrm{CC})$ phases were determined. Subsequently, Calbi et al. 2] have checked such a SFM by comparing its predictions with results provided by the nonlocal density-functional (NLDF) formulated by the Orsay-Paris (OP) collaboration [3] for superfluid helium. By looking at Figs. 15 and 16 in Ref. 2 one may realize that the $\mathrm{E} \rightarrow \mathrm{F}$ phase transitions given by SFM for ${ }^{4} \mathrm{He} / \mathrm{Li}$ and ${ }^{4} \mathrm{He} / \mathrm{Au}$ systems do not reproduce quite well results obtained from the OP-NLDF theory. In this report we suggest a correction which substantially improves the agreement.

For the descriptions provided in Refs. 1 and 2 it is assumed that ${ }^{4} \mathrm{He}$ atoms interact with the walls via the net potential

$$
U_{\text {slit }}(z)=V(z)+V(L-z),
$$

with $V(z)$ being the standard $(9,3)$ adsorption potential

$$
V(z)=\left[4 C_{3}^{3} /\left(27 D^{2}\right)\right] z^{-9}-C_{3} z^{-3},
$$

where $D$ is the well depth and $C_{3}$ is the strength of the asymptotic van der Waals interaction [4]. This potential $V(z)$ exhibits a minimum at

$$
z_{m}=\left[2 C_{3} /(3 D)\right]^{1 / 3} .
$$

A sample of parameters $D$ and $C_{3}$ corresponding to the interaction of ${ }^{4} \mathrm{He}$ atoms with several substrates given in Refs. 5 and 6 is listed in Table [1] The reader may find a plot of $D$ vs $C_{3}$ in Fig. 2 of Ref. 5 . It should be mentioned that for alkali metals the adsorption potentials of Ref. 6 are more attractive than the previous ones of Ref. 5 .

*Also at Carrera del Investigador Científico of the Consejo Nacional de Investigaciones Científicas y Técnicas, Av. Rivadavia 1917, RA1033 Buenos Aires, Argentina
TABLE I: Values of the well depth $D$, the van der Waals

\begin{tabular}{|c|c|c|c|c|c|c|c|c|}
\hline Surface & $\begin{array}{l}D^{a} \\
{[\mathrm{~K}]}\end{array}$ & $\begin{array}{r}C_{3}{ }^{a} \\
{\left[\mathrm{KA}^{3}\right]}\end{array}$ & $\begin{array}{c}z_{m} \\
{[\AA]}\end{array}$ & $D^{*}$ & $\overline{\sigma^{*}}$ & $\beta_{\mathrm{PH}}$ & $\beta_{\mathrm{HO}}$ & $\overline{\beta_{\mathrm{MHO}}}$ \\
\hline $\mathrm{Na}^{b}$ & 12.53 & 1197.4 & 3.99 & 8.03 & 0.2177 & & 2.65 & 2.18 \\
\hline $\mathrm{Li}^{b}$ & 17.87 & 1422.5 & 3.76 & 10.78 & 0.2054 & 2.80 & 2.81 & 2.53 \\
\hline $\mathrm{H}_{2}$ & 28.00 & 360.0 & 2.05 & 9.20 & 0.2487 & 2.23 & 2.32 & 2.34 \\
\hline $\mathrm{Mg}^{b}$ & 35.63 & 1850.2 & 3.26 & 18.64 & 0.1856 & 3.12 & 3.11 & 3.32 \\
\hline $\mathrm{Ne}$ & 60.00 & 163.0 & 1.22 & 11.74 & 0.2664 & 2.78 & 2.16 & 2.64 \\
\hline $\mathrm{Al}$ & 60.30 & 2340.0 & 2.96 & 28.63 & 0.1708 & 3.77 & 3.38 & 4.12 \\
\hline $\mathrm{Cu}$ & 63.80 & 2610.0 & 3.01 & 30.83 & 0.1669 & 3.90 & 3.46 & 4.28 \\
\hline $\mathrm{Ag}$ & 63.80 & 2890.0 & 3.11 & 31.90 & 0.1641 & 3.95 & 3.52 & 4.35 \\
\hline $\mathrm{NaCl}$ & 69.60 & 1230.0 & 2.28 & 25.42 & 0.1879 & 3.73 & 3.07 & 3.88 \\
\hline Ar & 78.90 & 870.0 & 1.94 & 24.63 & 0.1970 & 3.83 & 2.93 & 3.82 \\
\hline $\mathrm{Au}$ & 92.80 & 3180.0 & 2.84 & 42.27 & 0.1566 & 4.59 & 3.69 & 5.01 \\
\hline $\mathrm{LiF}$ & 94.00 & 1080.0 & 1.97 & 29.75 & 0.1872 & 4.26 & 3.08 & 4.20 \\
\hline $\mathrm{Gr}$ & 190.00 & 2130.0 & 1.96 & 59.64 & 0.1577 & 6.63 & 3.66 & 5.95 \\
\hline
\end{tabular}
strength $C_{3}$, the position of the potential's minimum $z_{m}$, the reduced well depth $D^{*}$ and other relevant quantities.

${ }^{a}$ Potential parameters $D$ and $C_{3}$ are taken from Ref. 5

except where noted. ${ }^{b}$ Potential parameters from Ref. 6

According to SFM, the $\mathrm{E} \rightarrow \mathrm{F}$ transition for films of thickness $\ell$ adsorbed on both walls of the slit is governed by the evolution of the reduced (i.e., in units of the surface tension $\sigma_{l v}=0.272 \mathrm{~K}^{-2}$ [7|) grand potential per unit area given by Eq. (8) of Ref. 1

$$
\begin{aligned}
\Omega_{\mathrm{F}}^{*}=\frac{\Omega_{\mathrm{F}}}{\sigma_{l v}}=4 & -D^{*}\left[g\{1+x\}+g\left\{L^{*}-1\right\}\right. \\
& \left.-g\left\{L^{*}-1-x\right\}\right]+2 x \Delta .
\end{aligned}
$$

Here the first term is the surface energy, the second is due to the helium-walls interaction, and the last one measures the departure from the saturated vapor pressure (SVP) condition. The reduced well depth and gap are

$$
\begin{gathered}
D^{*}=2 \rho_{0} D z_{m} / \sigma_{l v}, \\
\Delta=\left(\mu_{0}-\mu\right) \rho_{0} z_{m} / \sigma_{l v},
\end{gathered}
$$

where $\rho_{0}=0.021836 \AA^{-3}$ is the saturation equilibrium density and $\mu_{0}=-7.15 \mathrm{~K}$ the corresponding chemical 


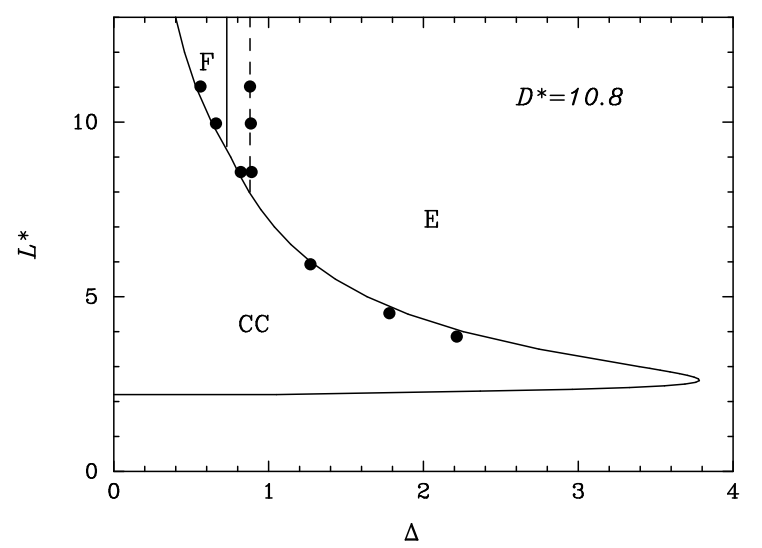

FIG. 1: Reduced phase diagram for ${ }^{4} \mathrm{He}$ confined by two planar walls of $\mathrm{Li}\left(D^{*}=10.78, z_{m}=3.76 \AA\right)$. Solid curves are SFM predictions and full circles OP-NLDF results, both of them calculated in the present work (see text). The dashed curve shows how much the correction introduced in the present work improves the prediction of the SFM for the $\mathrm{E} \rightarrow \mathrm{F}$ phase transition.

potential [7]. Furthermore,

$$
g\{y\}=\int_{1}^{y} d z^{*}\left[\frac{-U_{\text {slit }}\left(z_{m} z^{*}\right)}{D}\right]=\frac{11}{16}-\frac{3}{4} \frac{1}{y^{2}}+\frac{1}{16} \frac{1}{y^{8}},
$$

here all the reduced distances are given in terms of $z_{m}$

$$
z^{*}=z / z_{m}, \quad x=\ell / z_{m}, \quad \text { and } \quad L^{*}=L / z_{m},
$$

where $x$ is the film thickness that minimizes $\Omega_{\mathrm{F}}^{*}$.

Figure 1 shows that according to present calculations for the case of ${ }^{4} \mathrm{He}$ confined into slits of $\mathrm{Li}$ the reduced grand potential $\Omega_{\mathrm{F}}^{*}$ given by the SFM becomes negative for $L^{*}>9$ at $\Delta_{\mathrm{SFM}}=0.73$, while the OP-NLDF predicts that the $\mathrm{E} \rightarrow \mathrm{F}$ phase transition occurs at $\Delta_{\mathrm{DF}}=0.85$. Similar effects are also obtained for that substrates listed in Table \which are strong enough to produce a stable monolayer film. In order to illustrate this feature results for ${ }^{4} \mathrm{He} / \mathrm{Mg}$ and ${ }^{4} \mathrm{He} / \mathrm{Au}$ are plotted in Figs. 2 and 3 Let us recall that a slit of $\mathrm{Na}$ is too weak to produce a stable monolayer structure.

In Figs. 13 besides the $\mathrm{E} \rightarrow \mathrm{F}$ phase transition there are also indicated the $\mathrm{E} \rightarrow \mathrm{CC}$ and $\mathrm{F} \rightarrow \mathrm{CC}$ ones. These phase transitions are determined from comparisons with the corresponding grand potential per unit area obtained from Eq. (5) of Ref. 1, i.e.,

$$
\Omega_{\mathrm{CC}}^{*}=\frac{\Omega_{\mathrm{CC}}}{\sigma_{l v}}=2-D^{*} g\left\{L^{*}-1\right\}+\left(L^{*}-2\right) \Delta .
$$

In this work we shall demonstrate that the prediction given by the bare SFM is improved by introducing a correction to the surface term in the grand potential $\Omega_{\mathrm{F}}^{*}$ given by Eq. (4). An important shortcoming is that this term does not vanish in the limit of a zero-thickness film

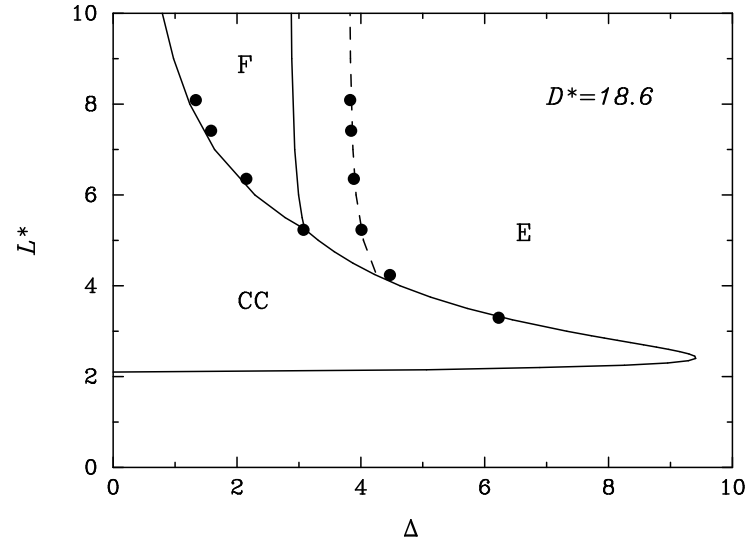

FIG. 2: Same as Fig. 1 but for ${ }^{4} \mathrm{He}$ confined by $\mathrm{Mg}\left(D^{*}=\right.$ $\left.18.64, z_{m}=3.26 \AA\right)$.

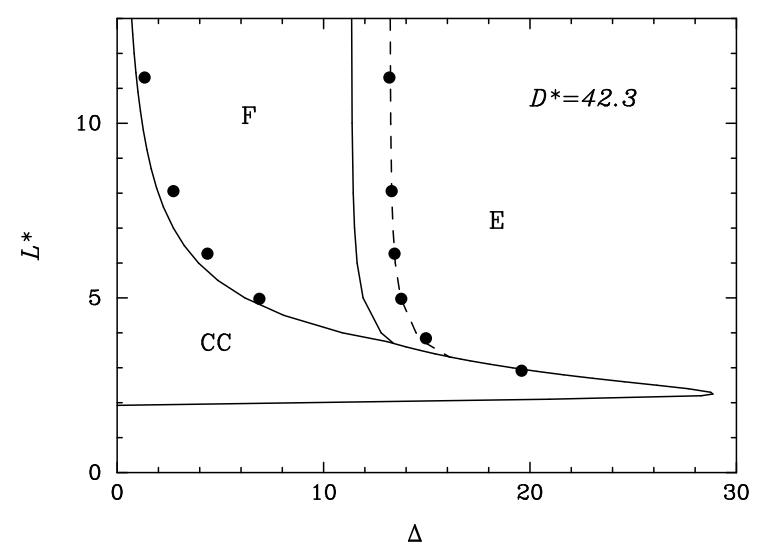

FIG. 3: Same as Fig. 11 but for ${ }^{4} \mathrm{He}$ confined by $\mathrm{Au}\left(D^{*}=\right.$ $42.27, z_{m}=2.84 \AA$ ).

(i.e. for $x=\ell / z_{m} \rightarrow 0$ ). In order to eliminate this failure one may follow the idea adopted by Cheng et al. [8] in writing their Eq. (2.4). So that, we shall assume that the surface contribution grows exponentially from zero at $\ell=0$ to the bare value 4 over a characteristic length $\zeta$

$$
\Omega_{\mathrm{F}}^{*}(\operatorname{surf})=4[1-\exp (-\ell / \zeta)]=4[1-\exp (-\beta x)],
$$

with

$$
\beta=z_{m} / \zeta=1 / \zeta^{*} .
$$

The parameter $\beta$ was determined for all the systems quoted in Table \by requiring that OP-NLDF results be reproduced by SFM. This means, we impose that the $\mathrm{E} \rightarrow$ $\mathrm{F}$ transition must occur at $\Delta_{\mathrm{DF}}$ for both models. These phenomenological values denoted $\beta_{\mathrm{PH}}$ are also included in Table【 The changes of the energetics due to the cutoff factor introduced in Eq. (10) only become important for rather thin films.

It should be also noted that, if a similar correction is included in the grand potential for the CC phase the 
corrected curves for the $\mathrm{E} \rightarrow \mathrm{CC}$ and $\mathrm{F} \rightarrow \mathrm{CC}$ transitions do not differ much from that displayed in Figs. 113

The following lines are devoted to propose a possible origin for the cut-off factor $\beta=z_{m} / \zeta$. As a first step, it is plausible to assume that the characteristic length $\zeta$ be related to the width of the ground-state (g.s.) wavefunction (submonolayer solution) of a simple harmonicoscillator approximation to the adsorption potential in the neighborhood of each wall

$$
V_{\text {app }}(z)=-D+V_{\mathrm{HO}}(z)=-D+\frac{1}{2} k\left(z-z_{m}\right)^{2},
$$

with the force constant

$$
k=\left[\frac{d^{2} V}{d z^{2}}\right]_{z=z_{m}}=18 C_{3}\left(\frac{3 D}{2 C_{3}}\right)^{5 / 3}=27 \frac{D}{z_{m}^{2}},
$$

see comment quoted as citation 44 in Ref. 8 . The dimensionless force constant becomes

$$
\kappa=k z_{m}^{2} / D=27 .
$$

So, the reduced version of $V_{\mathrm{app}}(z)$ reads

$$
V_{\mathrm{app}}^{*}\left(z^{*}\right)=\frac{V_{\mathrm{app}}\left(z_{m} z^{*}\right)}{D}=-1+\frac{27}{2}\left(z^{*}-1\right)^{2},
$$

and the reduced hamiltonian may be conveniently written as

$$
H_{\mathrm{HO}}^{*}=H_{\mathrm{app}}^{*}+1=-\frac{\hbar^{2}}{2 m_{\mathrm{eff}}} \frac{d^{2}}{d z^{* 2}}+\frac{27}{2}\left(z^{*}-1\right)^{2},
$$

with an effective helium mass

$$
m_{\mathrm{eff}}=m D z_{m}^{2} .
$$

The g.s. energy of $H_{\text {app }}^{*}$ is

$$
E_{\mathrm{app}}^{*}=-1+E_{\mathrm{HO}}^{*}=-1+\frac{1}{2} \frac{\hbar}{D} \sqrt{\frac{k}{m}}=-1+\frac{\sqrt{27 E_{k}^{*}}}{2},
$$

where $E_{k}^{*}=\hbar^{2} / m_{\text {eff }}$ is a dimensionless kinetic energy factor. Let us mention that as quoted in Ref. 9, for graphite ( $\mathrm{Gr}$ ) the semi-empirical He potential of Carlos and Cole 10, based on He scattering data, has $\kappa=26$. is

The probability density of the g.s. solution of Eq. (16)

$$
\left|\psi_{0}\left(z^{*}\right)\right|^{2}=\frac{1}{\sqrt{2 \pi} \sigma^{*}} e^{-\frac{1}{2}\left[\left(z^{*}-1\right) / \sigma^{*}\right]^{2}},
$$

with

$$
\sigma^{*}=\frac{\sigma}{z_{m}}=\frac{1}{z_{m}}\left(\frac{\hbar^{2}}{m k}\right)^{1 / 4}=\left(\frac{E_{k}^{*}}{27}\right)^{1 / 4} .
$$

Figure 4 shows examples of density profiles yielded by the DF theory for the lowest stable coverages. Let us recall that in the literature the surface thickness is defined as the distance over which the density falls off from the $90 \%$

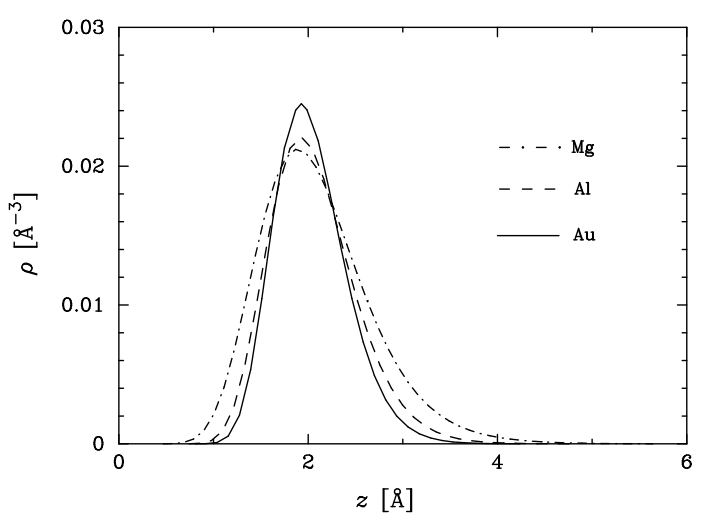

FIG. 4: Comparison of monolayer ${ }^{4} \mathrm{He}$ systems adsorbed on the left wall of broad slits of $\mathrm{Mg}, \mathrm{Al}$, and $\mathrm{Au}$. These films were determined at the $\mathrm{E} \rightarrow \mathrm{F}$ phase transition, i.e., they correspond to the lowest stable coverage in each case.

to $10 \%$ of the saturation value 11. In the case of the g.s. wavefunction given by Eq. (19) these quota lead to

$$
\begin{aligned}
& \mathcal{R}\left(\zeta_{i}^{*}\right)=\frac{\left|\psi_{0}\left(1+\zeta_{i}^{*}\right)\right|^{2}}{\left|\psi_{0}(1)\right|^{2}}=e^{-\frac{1}{2}\left[\zeta_{i}^{*} / \sigma^{*}\right]^{2}}=0.90, \\
& \mathcal{R}\left(\zeta_{f}^{*}\right)=\frac{\left|\psi_{0}\left(1+\zeta_{f}^{*}\right)\right|^{2}}{\left|\psi_{0}(1)\right|^{2}}=e^{-\frac{1}{2}\left[\zeta_{f}^{*} / \sigma^{*}\right]^{2}}=0.10 .
\end{aligned}
$$

From here one gets

$$
\begin{aligned}
\zeta^{*}=\zeta_{f}^{*}-\zeta_{i}^{*} & =\frac{4 \ln 3}{\zeta_{f}^{*}+\zeta_{i}^{*}}\left(\sigma^{*}\right)^{2}=\frac{2 \sqrt{2} \ln 3}{\sqrt{\ln 10}+\sqrt{\ln (10 / 9)}} \sigma^{*} \\
& =1.69 \sigma^{*} \simeq \sqrt{3} \sigma^{*} .
\end{aligned}
$$

It is interesting to notice that by assuming $\zeta^{*} \simeq \sqrt{3} \sigma^{*}$ the integral

$$
\mathcal{I}\left(\zeta^{*}\right)=\frac{1}{\sqrt{2 \pi} \sigma^{*}} \int_{1-\zeta^{*}}^{1+\zeta^{*}} d z^{*} e^{-\frac{1}{2}\left[\left(z^{*}-1\right) / \sigma^{*}\right]^{2}} \simeq 0.90,
$$

amounts about $90 \%$ of the total norm.

Turning to the cut-off parameter $\beta$, the expression derived from a pure harmonic oscillator analysis becomes

$$
\beta_{\mathrm{HO}}=\frac{1}{\zeta^{*}}=\frac{1}{\sqrt{3}}\left(\frac{27}{E_{k}^{*}}\right)^{1 / 4}=\left(\frac{3 m_{\mathrm{eff}}}{\hbar^{2}}\right)^{1 / 4} .
$$

The estimations provided by this formula are listed in Table [ From a glance at that table, one can realize that there is a very good agreement for the most weakly bounded monolayer systems, i.e., that confined by walls of $\mathrm{H}_{2}, \mathrm{Li}$ and $\mathrm{Mg}$. It is worthwhile to mention that the values of $\beta$ for ${ }^{4} \mathrm{He} / \mathrm{Li}$ and ${ }^{4} \mathrm{He} / \mathrm{Mg}$ are in the parameter region utilized by Cheng et al. [8] for showing how SFM results approach those given by a full NLDF theory in the case of adsorption on semi-infinite surfaces with planar 


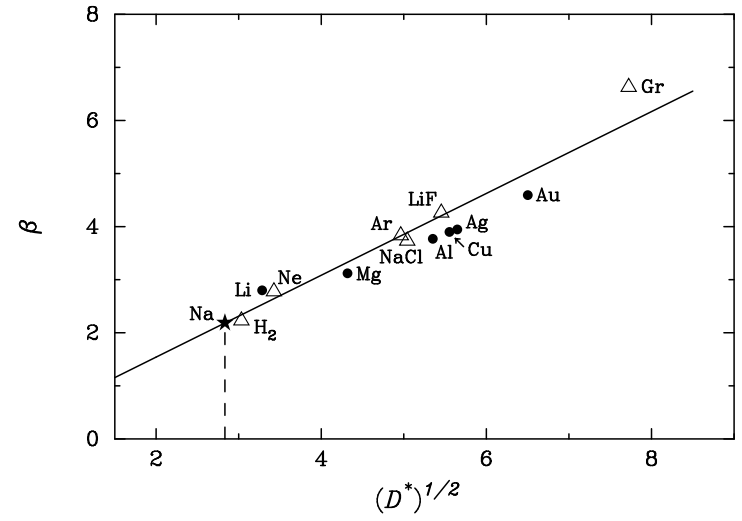

FIG. 5: Parameter $\beta_{\mathrm{PH}}$ obtained from equating SFM and DF results for ${ }^{4} \mathrm{He}$ films as a function of $\sqrt{D^{*}}$. Full circles are metallic substrates, while open triangles stand for other kind of materials. The star is the $\beta_{\mathrm{MHO}}$ value for a slit of Na. The solid straight line is given by Eq. (26).

geometry. In particular, $\beta=3$ is just the value chosen in that paper to illustrate the behavior.

The values of $\beta_{\mathrm{HO}}$ fall short if the helium atoms are exposed to stronger attractions. Hence, one could argue that a correction proportional to $D$ should be introduced if the harmonic oscillator approach for the potential $V(z)$ of Eq. (2) yields a g.s. oscillator energy much smaller than the well depth, i.e., when in Eq. (18) holds $\frac{1}{2} \frac{\hbar}{D} \sqrt{\frac{k}{m}} \ll 1$. In searching for such a dependence, it was realized that the values of $\beta_{\mathrm{PH}}$ become aligned when plotted as a function of $\sqrt{D^{*}}$. This behavior may be observed in Fig. [5] where $\mathrm{Na}$ indicates the threshold obtained for the formation of stable monolayer films. Similar results are obtained by using the more recent NLDF of Ref. 12 .

Therefore, as a next step the relative influence of effects due to the strength of the adsorption potential and to the resistance of helium atoms to be compressed was analyzed. It was found that if one modifies $\beta_{\mathrm{HO}}$ by including a factor which takes into account the ratio between the well depth $D$ and the compressibility at saturation density $1 / \rho_{0} \kappa_{v}=27.2 \mathrm{~K} \mathrm{[7]}$ in such a way that the total effect be proportional to $\sqrt{D^{*}}$

$$
\begin{aligned}
\beta_{\mathrm{MHO}} & =\beta_{\mathrm{HO}}\left(\frac{D}{1 / \rho_{0} \kappa_{v}}\right)^{1 / 4} \\
& =\left(\frac{3 m D z_{m}^{2}}{\hbar^{2}}\right)^{1 / 4} \times\left(\frac{D}{1 / \rho_{0} \kappa_{v}}\right)^{1 / 4} \\
& =\left(\frac{3}{4} \frac{m}{\hbar^{2}} \frac{\sigma_{l v}^{2}}{\rho_{0}} \kappa_{v}\right)^{1 / 4} \sqrt{D^{*}}
\end{aligned}
$$

one gets a good overall agreement as shown in Fig. 5 It is worthy of notice that the slope of this simple expression is written in terms of physical observables of bulk helium.

Moreover, it is important to emphasize that Fig. 5 collects data on substrates from $\mathrm{H}_{2}$ and $\mathrm{Li}$, which are barely able to support monolayer helium films, up to Gr (graphite), which is the strongest attractor for helium atoms [4]. Equation (26) can be considered as a nearly universal property since the straight line for $\beta_{\mathrm{MHO}}$ matches well the data of $\beta_{\mathrm{PH}}$ for all the substrates included in the present Table प i.e., for those plotted in Fig. 2 of Ref. 5 provided they ly above the dashed curve traced there. Notice that in that figure the points determined by $D$ and $C_{3}$ are spread over the whole drawing.

In conclusion, one can state that a simple semiphenomenological correction in the surface term of the SFM given by Eq. (4) yields an important improvement of the prediction for the $\mathrm{E} \rightarrow \mathrm{F}$ phase transition. The corrective term proposed in the present report contains a decaying exponential built by following the simple model formulated by Cheng et al. [8]. The cutoff parameter can be interpreted by taking into account properties of the harmonic g.s. wavefunction, the well depth of the adsorption potential and the compressibility of bulk helium.

\section{Acknowledgments}

This work was supported in part by the Ministry of Culture and Education of Argentina through Grants CONICET PIP No. 5138/05 and UBACyT No. X298.
[1] S. M. Gatica, M. M. Calbi, and M. W. Cole, Phys. Rev. E 59, 4484 (1999).

[2] M. M. Calbi, F. Toigo, S. M. Gatica, and M. W. Cole, Phys. Rev. B 60, 14935 (1999).

[3] J. Dupont-Roc, M. Himbert, N. Pavloff, and J. Treiner, J. Low Temp. Phys. 81, 31 (1990).

[4] L. W. Bruch, M. W. Cole, and E. Zaremba, Physical Adsorption: Forces and Phenomena (Oxford University Press, Oxford, 1997).

[5] E. Cheng, G. Ihn, and M. W. Cole, J. Low Temp. Phys. 14, 519 (1989).

[6] A. Chizmeshya, M. W. Cole, and E. Zaremba, J. Low
Temp. Phys. 110, 677 (1998).

[7] L. Szybisz, Eur. Phys. J. B 14, 733 (2000).

[8] E. Cheng, M. W. Cole, W. F. Saam, and J. Treiner, Phys. Rev. B 46, 13967 (1992).

[9] G. Vidali, M. W. Cole, and J. R. Klein, Phys. Rev. B 28, 3064 (1983).

[10] W. E. Carlos and M. W. Cole, Surf. Sci. 91, 339 (1980).

[11] L. Szybisz and I. Urrutia, Phys. Rev. B 68, 054518 (2003).

[12] F. Dalfovo, A. Lastri, L. Pricaupenko, S. Stringari, J. Treiner, Phys. Rev. B 52, 1193 (1995). 